\title{
Effect of inhaled drugs on anxiety and depression in patients with chronic obstructive pulmonary disease: a prospective observational study
}

This article was published in the following Dove Press journal:

International Journal of COPD

II April 2016

Number of times this article has been viewed

\author{
Min Kyung Hyun' \\ $\mathrm{Na}$ Rae Lee ${ }^{2}$ \\ Eun Jin Jang ${ }^{3}$ \\ Jae-Joon Yim ${ }^{4}$ \\ Chang-Hoon Lee \\ 'Department of Preventive Medicine, \\ College of Korean Medicine, Dongguk \\ University, Gyeongju, Republic of \\ Korea; ${ }^{2}$ National Evidence-based \\ Healthcare Collaborating Agency, \\ Seoul, Republic of Korea; ${ }^{3}$ Department \\ of Information Statistics, College of \\ Natural Science, Andong National \\ University, Andong, Republic of \\ Korea; ${ }^{4}$ Division of Pulmonary and \\ Critical Care Medicine, Department \\ of Internal Medicine, Seoul National \\ University College of Medicine, Seoul \\ National University Hospital, Seoul, \\ Republic of Korea
}

Correspondence: Chang-Hoon Lee Division of Pulmonary and Critical Care Medicine, Department of Internal Medicine, Seoul National University College of Medicine, Seoul National University Hospital, I0I Daehak-Ro, Jongno-Gu, Seoul 03080, Republic of Korea

Tel +82 220724743

Fax +8227629662

Email kauri670@empal.com
Objective: We investigated the effect of treatment with inhaled drugs on changes in mood, focusing on depression and anxiety during treatment in patients with newly diagnosed chronic obstructive pulmonary disease (COPD).

Methods: We prospectively selected new COPD patients from three Seoul National Hospitals. Participants underwent face-to-face interviews to evaluate clinical characteristics and drug use, and completed questionnaires using the Hospital Anxiety and Depression Scale (HADS) at the start of the project, the 4th week, and the 12th week. We compared changes in HADS scores both between inhaled corticosteroid (ICS)/long-acting $\beta_{2}$ agonist (LABA) users and non-ICS/ LABA users, as well as between long-acting muscarinic antagonist (LAMA) users and nonLAMA users. The general linear mixed model was applied for multivariate analysis.

Results: Ninety new COPD patients participated in the study. Of these 90 patients, 84 completed face-to-face interviews. In the univariate analysis, those who were treated by ICS/LABA or LAMA showed a significant increase in HADS-A at the 4-week evaluation $(0.73 \pm 2.56$, $P=0.038$ ), but the statistical significance was lost in the multivariable analysis. No significant difference was found in the change in HADS scores between ICS/LABA users and non-ICS/ LABA users, or between LAMA users and non-LAMA users in multivariate general linear mixed model analyses.

Conclusion: In this prospective observational study, we found no significant effects of inhaled treatment on mood problems among new COPD patients. Further research should be conducted to identify the association between anxiety and depression and inhaled drugs for COPD treatment.

Keywords: Hospital Anxiety and Depression Scale, mood disorders, adverse effects of inhaled drugs, chronic obstructive pulmonary disease

\section{Introduction}

Regular treatment with inhaled respiratory medications, including inhaled corticosteroids (ICSs), long-acting $\beta_{2}$ agonists (LABAs), and long-acting muscarinic antagonists (LAMAs), is well known to be the cornerstone in the management of chronic obstructive pulmonary disease (COPD). ${ }^{1}$ ICS and LABA drugs are prescribed widely in combination. The TORCH trial reported that treatment with this drug combination reduced exacerbations and improved the health status in COPD patients, ${ }^{2}$ as well as reduced the annual lung function decline rate in a post hoc analysis. ${ }^{3}$ The UPLIFT trial showed that LAMAs also improved symptoms, exercise tolerance, and health-related quality of life, and decreased exacerbations in COPD patients. ${ }^{4}$ 
However, inhaled treatment has adverse effects, including systemic complications such as diabetes mellitus, ${ }^{5}$ osteoporosis, ${ }^{6}$ fractures,${ }^{7}$ and risk of cardiovascular events. ${ }^{8,9}$ In addition, several studies have shown that $\beta$-adrenergic and cholinergic signaling are associated with anxiety and depression. ${ }^{10-13}$ Treatment with systemic corticosteroids for COPD could also affect mood problems, including hypomania, mania, depression, anxiety, panic disorder, and delirium. ${ }^{14,15}$ The latter finding suggests that inhaled drugs could also be associated with mood disorders. However, the impact of inhaled drugs on mood problems has rarely been evaluated. The purpose of this study was to evaluate the effect of treatment with inhaled drugs on changes in mood, focusing on depression and anxiety during treatment in patients with newly diagnosed COPD, using the Hospital Anxiety and Depression Scale (HADS), ${ }^{16}$ a widely used questionnaire on anxiety and depression in Korea. ${ }^{17-19}$

\section{Methods}

\section{Participants}

This prospective survey was conducted from August 1, 2011 to January 31, 2012, at three national hospitals: Seoul National University Hospital, Seoul Metropolitan Government-Seoul National University Boramae Medical Center, and Seoul National University Bundang Hospital. We prospectively selected new patients with COPD from outpatient clinics. New COPD patients were defined as follows: patients without inhaled medication within 3 months, and patients with postbronchodilator forced expiratory volume in 1 second/ forced vital capacity $<0.7 .{ }^{20}$ Participants received verbal and written information, and signed written consent was obtained for each participant. The two most common inhaled medications were ICS/LABAs and LAMAs. Therefore, we classified the patients into two groups depending on the ICS/ LABA prescribed (ICS/LABA vs non-ICS/LABA group) and LAMA prescribed (LAMA vs non-LAMA group), respectively. The study was approved by the Institutional Review Board of National Evidence-based Healthcare Collaborating Agency and all participating hospitals.

\section{Survey methods and questionnaires}

Face-to-face interviews were conducted with participants using the standard HADS questionnaire by well-trained nurses. However, when face-to-face interviews were not possible during the course of treatment, telephone surveys were conducted instead. HADS was originally developed to detect states of depression and anxiety in the hospital's outpatient clinic. ${ }^{16}$ It is a selfassessment procedure comprising 14 questions, of which seven are anxiety-related questions (HADS-A) and the remainder are depression-related questions (HADS-D). Each item was scored between 0 and 3 points; each patient was scored from 0 to 21 for either anxiety or depression, with higher scores signifying a higher level of depression or anxiety. ${ }^{21}$

\section{Statistical analysis}

Chi-squared test and Fisher's exact test were used to compare the differences between ICS/LABAs and non-ICS/LABAs, and between LAMAs and non-LAMAs, in different subsets of patients. The various scores compiled at the start of the project, the 4th week, and the 12th week were calculated, and the change in scores was presented as the mean, standard deviation (SD), median, 25th percentile (Q1), and 75th percentile (Q3). The Wilcoxon signed-rank test was used to compare the depression or anxiety levels before and after the inhaled therapy. For multivariate analysis, we applied the general linear mixed model. In this study, a two-sided $P$-value $<0.05$ was considered statistically significant. All statistical analyses were performed using SAS version 9.1.3 (SAS Institute Inc, Cary, NC, USA).

\section{Results \\ Baseline characteristics}

Ninety newly diagnosed COPD patients were enrolled. Among these 90 patients, four patients were excluded because they had received inhaled medication due to respiratory problems within the last 3 months, and two patients did not complete the HADS questionnaire. Finally, 84 patients were included in the analyses, and they consisted of 31 patients with mild COPD (GOLD 1), 41 patients with moderate COPD (GOLD 2), nine patients with severe COPD (GOLD 3), and three patients whose COPD status was unknown. The details given in Table 1 show that demographic characteristics and sex (males comprised $77.38 \%$ ) were similar between the ICS/LABA and non-ICS/LABA groups, and between the LAMA and nonLAMA groups. Table 2 lists the clinical characteristics of the participants. The LAMA group had a lower body mass index $(P=0.011)$ and lower forced expiratory volume in 1 second than the non-LAMA group $(1.59 \pm 0.45 \mathrm{~L}$ vs $2.16 \pm 0.82 \mathrm{~L}$; $P=0.001)$. Other clinical characteristics were not significantly different among the groups. Approximately $6 \%$ of the participants had a history of either anxiety or depression, and $2.38 \%$ of them underwent antianxiety or antidepressive treatment.

\section{Change in HADS scores}

Table 3 lists the crude changes in HADS scores according to time in the univariate analysis (Figure 1). 
Table I Demographic characteristics of the participants

\begin{tabular}{|c|c|c|c|c|c|c|c|}
\hline \multirow[t]{2}{*}{ Characteristics } & Total $(\mathbf{N}=\mathbf{8 4})$ & $\begin{array}{l}\text { ICS/LABA } \\
(\mathrm{N}=35)\end{array}$ & $\begin{array}{l}\text { Non-ICS/LABA } \\
(\mathrm{N}=49)\end{array}$ & $P$-value ${ }^{a}$ & $\begin{array}{l}\text { LAMA } \\
(\mathrm{N}=3 \mathrm{I})\end{array}$ & $\begin{array}{l}\text { Non-LAMA } \\
(\mathrm{N}=53)\end{array}$ & $P$-value ${ }^{a}$ \\
\hline & $\mathbf{N}(\%)$ & $\mathbf{N}(\%)$ & $\mathbf{N}(\%)$ & & $\mathbf{N}(\%)$ & $\mathbf{N}(\%)$ & \\
\hline Sex & & & & 0.965 & & & 0.030 \\
\hline Male & $65(77.38)$ & $27(77.14)$ & $38(77.55)$ & & $28(90.32)$ & $37(69.8 I)$ & \\
\hline Female & $19(22.62)$ & $8(22.86)$ & II (22.45) & & $3(9.68)$ & $16(30.19)$ & \\
\hline Age (years) & & & & 0.807 & & & 0.329 \\
\hline Mean \pm SD & $63.67 \pm 13.20$ & $64.09 \pm 12.13$ & $63.37 \pm 14.03$ & 0.467 & $65.52 \pm 11.44$ & $62.58 \pm 14.12$ & 0.608 \\
\hline$<50$ & $9(10.7 I)$ & $2(5.7 \mathrm{I})$ & $7(14.29)$ & & $3(9.68)$ & $6(11.32)$ & \\
\hline $50-59$ & $20(23.8 I)$ & $9(25.7 \mathrm{I})$ & II (22.45) & & $5(16.13)$ & $15(28.30)$ & \\
\hline $60-69$ & $19(22.62)$ & $10(28.57)$ & $9(18.37)$ & & $8(25.8 \mathrm{I})$ & II (20.75) & \\
\hline$\geq 70$ & $36(42.86)$ & $14(40.00)$ & $22(44.90)$ & & $15(48.39)$ & $21(39.62)$ & \\
\hline Marital status & & & & 0.112 & & & 0.365 \\
\hline Single & $15(17.86)$ & $9(25.7 \mathrm{I})$ & $6(12.24)$ & & $4(12.90)$ & II (20.75) & \\
\hline Married & $69(82.14)$ & $26(74.29)$ & $43(87.76)$ & & $27(87.10)$ & $42(79.25)$ & \\
\hline Education & & & & 0.371 & & & 0.296 \\
\hline Less than middle school & $36(42.86)$ & $17(48.57)$ & $19(38.78)$ & & II (35.48) & $25(47.17)$ & \\
\hline High school or beyond & $48(57.14)$ & I8 (5I.43) & $30(6 \mid .22)$ & & $20(64.52)$ & $28(52.83)$ & \\
\hline Job status & & & & 0.817 & & & 0.613 \\
\hline Employed & $54(64.29)$ & $22(62.86)$ & $32(65.3 I)$ & & $21(67.74)$ & $33(62.26)$ & \\
\hline Unemployed & $30(35.7 I)$ & $13(37.14)$ & $17(34.69)$ & & $10(32.26)$ & $20(37.74)$ & \\
\hline Income & & & & 0.768 & & & 0.311 \\
\hline$\leq 2$ million $\mathrm{KRW} /$ month & $44(52.38)$ & $19(54.29)$ & $25(51.02)$ & & $14(45.16)$ & $30(56.60)$ & \\
\hline$>2$ million $\mathrm{KRW} /$ month & $40(47.62)$ & $16(45.7 \mid)$ & $24(48.98)$ & & $17(54.84)$ & $23(43.40)$ & \\
\hline
\end{tabular}

Note: ${ }^{\mathrm{a}} \chi^{2}$ test.

Abbreviations: ICS, inhaled corticosteroid; LABA, long-acting $\beta_{2}$ agonist; LAMA, long-acting muscarinic antagonist; SD, standard deviation.

\section{Total}

At baseline, the mean total HADS score of the 84 patients was $9.75 \pm 5.30$ before starting inhaled medication. Four weeks after inhaled medication, the mean total HADS score was $10.72 \pm 6.68$, and the change from baseline was $1.24 \pm 4.67$ $(P=0.065)$. After 12 weeks, the mean total HADS score was $10.95 \pm 6.25$, and the change from baseline was $1.63 \pm 5.34$ $(P=0.058)$. After 4 weeks, the anxiety scores (HADS-A) significantly increased by $0.73 \pm 2.56(P=0.038)$, and there was no statistically significant change between baseline and the 12 th week $(0.72 \pm 2.84 ; P=0.100)$. In addition, no significant statistical change was observed in the depression scores (HADS-D).

\section{ICS/LABA vs non-ICS/LABA group}

In the ICS/LABA group, no statistically significant change was observed in HADS score between baseline and the 4th and 12 th weeks. However, in the non-ICS/LABA group, 4 weeks after inhaled medication, the mean total HADS score was $11.23 \pm 6.86$, and the change from baseline was statistically significant $(2.03 \pm 4.82 ; P=0.008)$. After 4 weeks, the HADS-A significantly increased by $1.03 \pm 2.44(P=0.013)$, and the HADS-D significantly increased by $1.00 \pm 2.87$ $(P=0.031)$. No significant statistical change was found between baseline and the 12 th week. The ICS/LABA group showed a relatively better effect on the change in the total HADS score between baseline and 4 weeks than that in the non-ICS/LABA group, but the difference was not statistically significant $(P=0.055)$. In multivariate analysis, ICS/LABA use tended to decrease the HADS scores compared to nonICS/LABA use, but the difference did not reach statistical significance (Table 4).

\section{LAMA vs non-LAMA group}

In the LAMA and non-LAMA groups, the HADS scores were not statistically significant between baseline and the 4 th and 12 th weeks. After 12 weeks, the mean total HADS score change from baseline was $1.83 \pm 4.86(P=0.064)$ in the LAMA group, and the HADS-A score change from baseline was $1.12 \pm 2.76(P=0.065)$. No statistically significant difference was observed in the change in HADS score between the LAMA and non-LAMA groups. Multivariate analysis showed that LAMA use did not lead to a statistically significant increase in HADS score (Table 4).

\section{Discussion}

To the best of our knowledge, this is the first prospective study conducted to elucidate whether inhaled treatment 
Table 2 Clinical characteristics of the participants

\begin{tabular}{|c|c|c|c|c|c|c|c|}
\hline \multirow[t]{2}{*}{ Characteristics } & \multirow{2}{*}{$\begin{array}{l}\begin{array}{l}\text { Total } \\
(\mathbf{N}=84)\end{array} \\
\mathbf{N}(\%)\end{array}$} & \multirow{2}{*}{$\begin{array}{l}\begin{array}{l}\text { ICS/LABA } \\
(\mathrm{N}=35) \\
\mathrm{N}(\%)\end{array}\end{array}$} & \multirow{2}{*}{$\begin{array}{l}\text { Non-ICS/LABA } \\
(\mathbf{N}=49) \\
\mathbf{N}(\%)\end{array}$} & \multirow[t]{2}{*}{$P$-value ${ }^{a}$} & \multirow{2}{*}{$\begin{array}{l}\text { LAMA } \\
(\mathbf{N}=3 I) \\
\mathbf{N}(\%)\end{array}$} & \multirow{2}{*}{$\begin{array}{l}\text { Non-LAMA } \\
(\mathrm{N}=53) \\
\mathbf{N}(\%)\end{array}$} & \multirow[t]{2}{*}{$P$-value ${ }^{a}$} \\
\hline & & & & & & & \\
\hline \multicolumn{8}{|l|}{ BMI $(n=82)$} \\
\hline$\leq 18.5$ & $10(12.20)$ & $4(11.76)$ & $6(12.50)$ & 0.715 & $8(3.33)$ & $2(3.92)$ & 0.011 \\
\hline $18.5-24.9$ & $49(59.76)$ & $22(64.7 I)$ & $27(56.25)$ & & $17(73.33)$ & $32(62.75)$ & \\
\hline$\geq 25.0$ & $23(28.05)$ & $8(23.53)$ & $15(31.25)$ & & $6(20.00)$ & $17(33.33)$ & \\
\hline \multicolumn{8}{|l|}{ Pulmonary function test } \\
\hline \multicolumn{8}{|l|}{$\mathrm{FEV}_{1}(\mathrm{n}=8 \mathrm{I})$} \\
\hline L, Mean \pm SD & $1.94 \pm 0.75$ & $1.94 \pm 0.74$ & $1.94 \pm 0.77$ & 0.997 & $1.59 \pm 0.45$ & $2.16 \pm 0.82$ & 0.001 \\
\hline$\%$, Mean \pm SD & $76.36 \pm 24.75$ & $76.91 \pm 27.80$ & $75.98 \pm 22.72$ & 0.869 & $61.81 \pm 13.64$ & $85.38 \pm 25.87$ & $<0.000$ I \\
\hline \multicolumn{8}{|l|}{ FVC $(n=78)$} \\
\hline$L$, Mean \pm SD & $3.33 \pm 0.91$ & $3.31 \pm 0.92$ & $3.34 \pm 0.92$ & 0.862 & $3.11 \pm 0.74$ & $3.48 \pm 0.99$ & 0.082 \\
\hline$\%$, Mean \pm SD & $92.72 \pm 22.29$ & $91.35 \pm 20.89$ & $93.62 \pm 23.35$ & 0.664 & $87.48 \pm 26.01$ & $96.17 \pm 18.97$ & 0.092 \\
\hline \multicolumn{8}{|l|}{ Smoking } \\
\hline Never smoked & $22(26.19)$ & $9(25.7 \mathrm{I})$ & $13(26.53)$ & 0.264 & $4(12.90)$ & $18(33.96)$ & 0.101 \\
\hline Past smoker & $47(55.95)$ & $18(48.57)$ & $30(61.22)$ & & $21(67.74)$ & $26(49.06)$ & \\
\hline Currently smoking & $15(17.86)$ & $9(25.7 \mathrm{I})$ & $6(12.24)$ & & $6(19.35)$ & $9(16.98)$ & \\
\hline \multicolumn{8}{|l|}{ Pack year $(n=8 I)$} \\
\hline Mean \pm SD & $28.13 \pm 29.36$ & $24.46 \pm 20.17$ & $30.64 \pm 34.27$ & 0.312 & $32.94 \pm 21.50$ & $25.14 \pm 33.18$ & 0.203 \\
\hline \multicolumn{8}{|c|}{ Secondhand smoke (home) } \\
\hline No & $57(67.86)$ & $21(60.00)$ & $36(73.47)$ & 0.344 & $23(74.19)$ & $34(64.15)$ & 0.636 \\
\hline Sometimes & $17(20.24)$ & $8(22.86)$ & $9(18.37)$ & & $5(16.13)$ & $12(22.64)$ & \\
\hline Everyday & $10(11.90)$ & $6(17.14)$ & $4(8.16)$ & & $3(9.68)$ & $7(13.21)$ & \\
\hline \multicolumn{8}{|c|}{ Secondhand smoke (office) $(n=83)$} \\
\hline No & $53(63.86)$ & $22(64.7 I)$ & $31(63.27)$ & 0.980 & $19(63.33)$ & $34(64.15)$ & 0.462 \\
\hline Sometimes & $18(21.69)$ & $7(20.59)$ & II (22.45) & & $5(16.67)$ & $13(24.53)$ & \\
\hline Everyday & $12(14.46)$ & $5(\mid 4.7 I)$ & $7(14.29)$ & & $6(20.00)$ & $6(11.32)$ & \\
\hline \multicolumn{8}{|c|}{ Period of secondhand smoke (years) } \\
\hline $0-1$ & $21(25.00)$ & $10(28.57)$ & II (22.45) & 0.645 & $5(16.13)$ & $16(30.19)$ & 0.164 \\
\hline $1-9$ & $14(16.67)$ & $4(11.43)$ & $10(20.4 I)$ & & $3(9.68)$ & II (20.75) & \\
\hline $10-29$ & $28(33.33)$ & II (3I.43) & $17(34.69)$ & & $13(4 \mid .94)$ & $15(28.30)$ & \\
\hline$\geq 30$ & $21(25.00)$ & $10(28.57)$ & II (22.45) & & $10(32.26)$ & II (20.75) & \\
\hline \multicolumn{8}{|c|}{ Past diagnosis of anxiety or depression } \\
\hline Yes & $5(5.95)$ & I (2.86) & $4(8.16)$ & $0.396^{\mathrm{b}}$ & $2(6.45)$ & $3(5.66)$ & $1.000^{\mathrm{b}}$ \\
\hline No & $79(94.05)$ & $34(97.14)$ & $45(91.84)$ & & $29(93.55)$ & $50(94.34)$ & \\
\hline \multicolumn{8}{|c|}{ Current treatment of anxiety or depression } \\
\hline Yes & $2(2.38)$ & I (2.86) & I (2.04) & $1.000^{\mathrm{b}}$ & I (3.23) & I (I.89) & $1.000^{\mathrm{b}}$ \\
\hline No & $82(97.62)$ & $34(97.14)$ & $48(97.96)$ & & $30(96.77)$ & $52(98.11)$ & \\
\hline
\end{tabular}

Notes: ${ }^{\mathrm{a}} \chi^{2}$ test; ${ }^{\mathrm{b}}$ Fisher's exact test.

Abbreviations: BMI, body mass index; $\mathrm{FEV}_{1}$, forced expiratory volume in I second; FVC, forced vital capacity; ICS, inhaled corticosteroid; LABA, long-acting $\beta_{2}$ agonist; LAMA, long-acting muscarinic antagonist; SD, standard deviation.

among COPD patients has an effect on anxiety and depression. The results showed that inhaled ICS/LABA or inhaled LAMA had no significant effect on the HADS score in newly diagnosed COPD patients.

It has been considered that inhaled drugs for COPD treatment may affect mood problems. In fact, major drug categories for COPD treatment could be associated with mood problems. Activation of $\beta$-adrenoceptors in the bed nucleus of the stria terminalis by $\beta$-agonists induced anxiety-like behavior in rats in a dose-dependent manner. ${ }^{10}$
Depression and related structural and functional changes in brain areas are thought to be associated with cholinergic dysfunction. ${ }^{10,13}$ Furthermore, in principle, anticholinergics can elevate heart rate, a finding that could contribute to an increase in anxiety. In fact, it was reported that blockade of muscarinic receptors in the central nervous system can cause anxiety and agitation in patients with anticholinergic overdose. ${ }^{22} \beta$-agonists, including new, selective $\beta_{2}$-agonists (salmeterol and formoterol), increase heart rate. ${ }^{23-25}$ Anticholinergic muscarinic $\left(\mathrm{M}_{3}\right)$ receptor antagonists also 
Table 3 HADS questionnaire scores

\begin{tabular}{|c|c|c|c|c|c|c|c|c|c|}
\hline \multirow[t]{3}{*}{ HADS scores } & \multirow{3}{*}{$\begin{array}{l}\frac{\text { Baseline }}{\text { Mean } \pm S D} \\
\text { Median } \\
(q \mathrm{q}, \mathrm{q} 3)\end{array}$} & \multirow{3}{*}{$\begin{array}{l}\frac{4 \text { weeks }}{\text { Mean } \pm S D} \\
\text { Median } \\
(q \mathrm{q}, \mathrm{q} 3)\end{array}$} & \multicolumn{2}{|c|}{ Change from baseline } & \multirow[t]{3}{*}{$P$-value ${ }^{b}$} & \multirow{3}{*}{$\begin{array}{l}\frac{12 \text { weeks }}{\text { Mean } \pm \text { SD }} \\
\text { Median } \\
(\mathrm{ql}, \mathrm{q} 3)\end{array}$} & \multicolumn{2}{|c|}{ Change from baseline } & \multirow[t]{3}{*}{$P$-value ${ }^{b}$} \\
\hline & & & Mean \pm SD & $P$-value ${ }^{a}$ & & & Mean \pm SD & $P$-value ${ }^{a}$ & \\
\hline & & & \multicolumn{2}{|l|}{$\begin{array}{l}\text { Median } \\
(q 1, q 3)\end{array}$} & & & \multicolumn{2}{|l|}{$\begin{array}{l}\text { Median } \\
(q \mathrm{q}, \mathrm{q} 3)\end{array}$} & \\
\hline Total & $(\mathrm{N}=84)$ & $(\mathrm{N}=67)$ & $(\mathrm{N}=67)$ & & & $(\mathrm{N}=55)$ & $(\mathrm{N}=54)$ & & \\
\hline \multirow[t]{2}{*}{ Total score } & $9.75 \pm 5.30$ & $10.72 \pm 6.68$ & $1.24 \pm 4.67$ & 0.065 & & $10.95 \pm 6.25$ & $1.63 \pm 5.34$ & 0.058 & \\
\hline & $9.00(6,13)$ & $10.00(7,14)$ & $0.00(-1,3)$ & & & $10.00(7,15)$ & I.50 $(-2,5)$ & & \\
\hline \multirow[t]{2}{*}{ Anxiety } & $3.92 \pm 2.79$ & $4.63 \pm 3.76$ & $0.73 \pm 2.56$ & 0.038 & & $4.5 \mathrm{I} \pm 3.35$ & $0.72 \pm 2.84$ & 0.100 & \\
\hline & $3.50(2,6)$ & $4.00(2,7)$ & $0.00(-1, I)$ & & & $4.00(2,7)$ & $0.00(-1,3)$ & & \\
\hline \multirow[t]{2}{*}{ Depression } & $5.83 \pm 3.13$ & $6.09 \pm 3.41$ & $0.5 I \pm 2.7 I$ & 0.171 & & $6.44 \pm 3.56$ & $0.91 \pm 3.26$ & 0.061 & \\
\hline & $6.00(3,8)$ & $6.00(4,8)$ & $0.00(-1,2)$ & & & $6.00(4,9)$ & $0.50(-1,2)$ & & \\
\hline ICS/LABA & $(\mathrm{N}=35)$ & $(\mathrm{N}=28)$ & $(\mathrm{N}=28)$ & & & $(\mathrm{N}=20)$ & $(\mathrm{N}=19)$ & & \\
\hline \multirow[t]{3}{*}{ Total score } & $10.14 \pm 5.30$ & $10.00 \pm 6.46$ & $0.14 \pm 4.30$ & 0.878 & 0.055 & $11.05 \pm 4.63$ & $1.26 \pm 5.43$ & 0.504 & 0.723 \\
\hline & $10.00(7,14)$ & $8.50(7,12.5)$ & $0.00(-2,2)$ & & & 10.50 & $1.00(-3,3)$ & & \\
\hline & & & & & & $(8,14.5)$ & & & \\
\hline \multirow[t]{2}{*}{ Anxiety } & $4.26 \pm 2.94$ & $4.54 \pm 3.95$ & $0.32 \pm 2.70$ & 0.809 & 0.125 & $4.55 \pm 2.65$ & $0.42 \pm 3.10$ & 0.735 & 0.465 \\
\hline & $5.00(1,6)$ & $4.50(2,7)$ & $0.00(-I, I)$ & & & $4.00(2,6.5)$ & $-0.50(3,9)$ & & \\
\hline \multirow[t]{3}{*}{ Depression } & $5.89 \pm 3.12$ & $5.46 \pm 3.14$ & $-0.18 \pm 2.36$ & 0.656 & 0.098 & $6.50 \pm 3.22$ & $0.84 \pm 3.37$ & 0.366 & 0.993 \\
\hline & $5.00(3,9)$ & $5.00(3,7)$ & 0.00 & & & $6.50(4,9)$ & $1.00(-2,3)$ & & \\
\hline & & & $(-1.5,0.5)$ & & & & & & \\
\hline Non-ICS/LABA & $(\mathrm{N}=49)$ & $(\mathrm{N}=39)$ & $(\mathrm{N}=39)$ & & & $(\mathrm{N}=35)$ & $(\mathrm{N}=35)$ & & \\
\hline \multirow[t]{2}{*}{ Total score } & $9.47 \pm 5.34$ & $11.23 \pm 6.86$ & $2.03 \pm 4.82$ & 0.008 & & $10.89 \pm 7.08$ & $1.83 \pm 5.35$ & 0.073 & \\
\hline & $9.00(5,13)$ & $10.00(7,15)$ & $1.00(0,4)$ & & & $9.00(6,15)$ & $2.00(-2,5)$ & & \\
\hline \multirow[t]{2}{*}{ Anxiety } & $3.67 \pm 2.68$ & $4.69 \pm 3.67$ & $1.03 \pm 2.44$ & 0.013 & & $4.49 \pm 3.74$ & $0.89 \pm 2.72$ & 0.067 & \\
\hline & $3.00(2,5)$ & $4.00(2,7)$ & $1.00(0,2)$ & & & $5.00(I, 7)$ & $0.00(-1,3)$ & & \\
\hline \multirow[t]{2}{*}{ Depression } & $5.80 \pm 3.18$ & $6.54 \pm 3.56$ & $1.00 \pm 2.87$ & 0.031 & & $6.40 \pm 3.78$ & $0.94 \pm 3.24$ & 0.087 & \\
\hline & $6.00(3,8)$ & $6.00(5,8)$ & $0.00(0,2)$ & & & $6.00(4,9)$ & $0.00(-1,2)$ & & \\
\hline LAMA & $(\mathrm{N}=3 \mathrm{I})$ & $(\mathrm{N}=27)$ & $(\mathrm{N}=27)$ & & & $(\mathrm{N}=24)$ & $(\mathrm{N}=24)$ & & \\
\hline \multirow[t]{3}{*}{ Total score } & $8.58 \pm 5.57$ & $10.19 \pm 7.93$ & $1.63 \pm 5.13$ & 0.112 & 0.597 & $10.88 \pm 7.48$ & $1.83 \pm 4.86$ & 0.064 & 0.523 \\
\hline & $8.00(3,12)$ & $9.00(3,14)$ & $1.00(-1,3)$ & & & $9.50(6,15)$ & 2.00 & & \\
\hline & & & & & & & $(-1.5,5)$ & & \\
\hline \multirow[t]{2}{*}{ Anxiety } & $3.32 \pm 2.59$ & $4.26 \pm 4.07$ & $1.00 \pm 2.75$ & 0.124 & 0.654 & $4.58 \pm 4.10$ & $1.12 \pm 2.76$ & 0.065 & 0.334 \\
\hline & $3.00(2,5)$ & $3.00(1,7)$ & $1.00(-1, I)$ & & & $4.50(1.5,7)$ & $1.00(-1,3)$ & & \\
\hline \multirow[t]{2}{*}{ Depression } & $5.26 \pm 3.30$ & $5.93 \pm 4.10$ & $0.63 \pm 2.76$ & 0.212 & 0.636 & $6.29 \pm 3.83$ & $0.7 I \pm 2.44$ & 0.105 & 0.806 \\
\hline & $5.00(3,8)$ & $6.00(2.8)$ & $0.00(-1,2)$ & & & $6.00(3,8)$ & $1.00(-1,2)$ & & \\
\hline Non-LAMA & $(\mathrm{N}=53)$ & $(\mathrm{N}=40)$ & $(\mathrm{N}=40)$ & & & $(\mathrm{N}=23)$ & $(\mathrm{N}=23)$ & & \\
\hline \multirow[t]{3}{*}{ Total score } & $10.43 \pm 5.07$ & $11.08 \pm 5.76$ & $0.98 \pm 4.39$ & 0.279 & & $11.00 \pm 5.24$ & I. $.47 \pm 5.77$ & 0.349 & \\
\hline & $10.00(7,14)$ & 10.00 & $0.00(-2,2)$ & & & $10.00(8,14)$ & $0.50(-3,3)$ & & \\
\hline & & $(7,14.5)$ & & & & & & & \\
\hline Anxiety & $4.26 \pm 2.87$ & $4.88 \pm 3.57$ & $0.55 \pm 2.43$ & 0.194 & & $4.45 \pm 2.72$ & $0.40 \pm 2.91$ & 0.513 & \\
\hline & $4.00(2,6)$ & $4.50(2,7)$ & 0.00 & & & $4.00(2,7)$ & $0.00(-1,2)$ & & \\
\hline & & & $(-0.5,1.5)$ & & & & & & \\
\hline Depression & $6.17 \pm 3.01$ & $6.20 \pm 2.90$ & $0.43 \pm 2.71$ & 0.433 & & $6.55 \pm 3.39$ & $1.07 \pm 3.82$ & 0.253 & \\
\hline & $6.00(4,9)$ & $6.00(4,7.5)$ & $0.00(-I, I)$ & & & $6.00(4,9)$ & $0.00(-1,2)$ & & \\
\hline
\end{tabular}

Notes: Change $=4$ weeks - baseline or 12 weeks - baseline. ${ }^{a}$ Wilcoxon signed-rank test for the change in scores from baseline to 4 and 12 weeks. ${ }^{b}$ Wilcoxon signed-rank test for the change in score from baseline to 4 and 12 weeks in the ICS/LABA vs non-ICS/LABA as well as LAMA vs non-LAMA. Anxiety: item-I,3,5,7,9,II,I3; depression: item-2,4,6,8,10,12,14.

Abbreviations: HADS, Hospital Anxiety and Depression Scale; ICS, inhaled corticosteroid; LABA, long-acting $\beta_{2}$ agonist; LAMA, long-acting muscarinic antagonist; SD, standard deviation.

inhibit parasympathetic control of heart rate..$^{26,27}$ Corticosteroids can also affect mood problems, including hypomania, mania, depression, anxiety, panic disorder, and delirium. ${ }^{14,15}$ A significantly higher self-reported depression score was reported in COPD patients receiving $20-40 \mathrm{mg} /$ day systemic prednisone for $10-14$ days. ${ }^{28}$
Anxiety and depression are important comorbidities of COPD, with prevalences estimated to be $2 \%-80 \%$ and $7 \%-80 \%$, respectively $;{ }^{29}$ findings that could be associated with cigarette smoke exposure, physical inactivity, social isolation, multiple episodes of dyspnea, and chronic hypoxia. ${ }^{30}$ These mood disorders, particularly depression, are associated with 


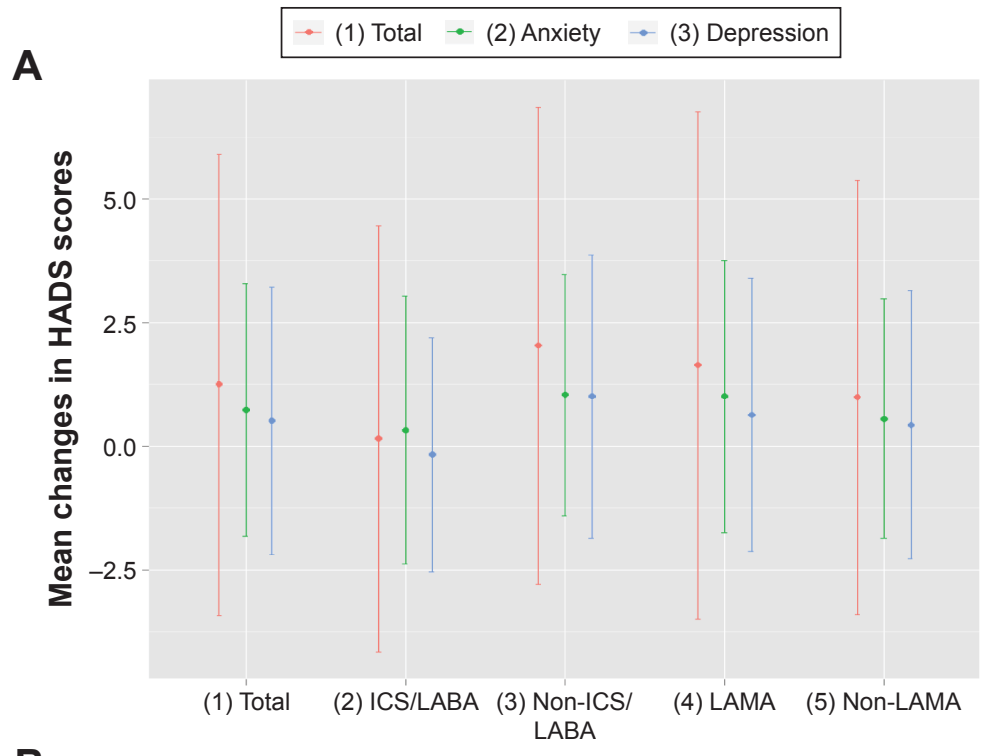

B

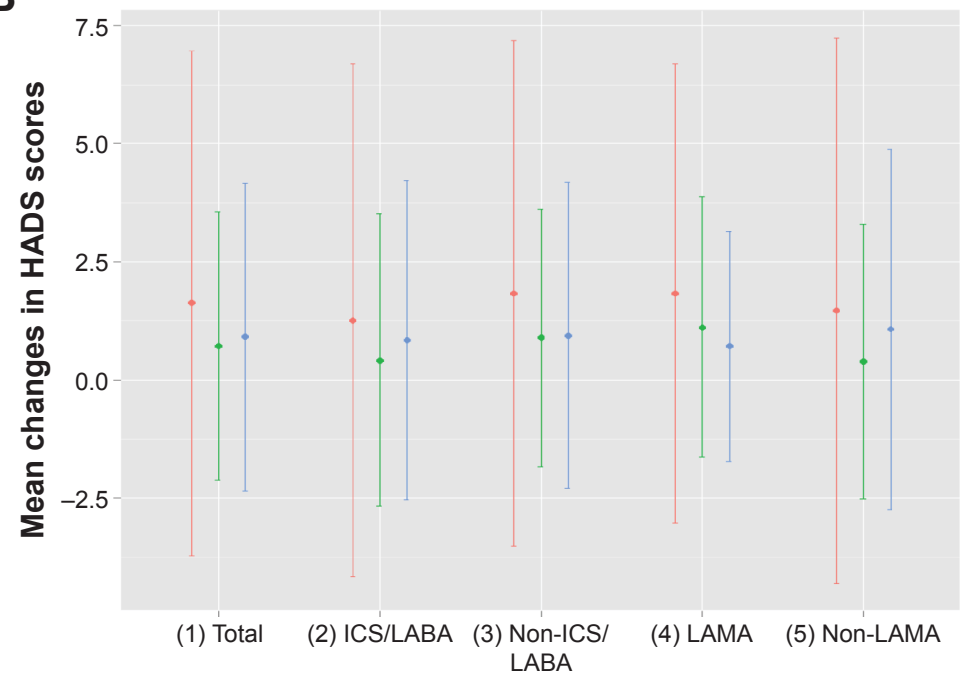

Figure I The mean changes in HADS scores from baseline to (A) 4 weeks and (B) 12 weeks.

Abbreviations: HADS, Hospital Anxiety and Depression Scale; ICS, inhaled corticosteroid; LABA, long-acting $\beta_{2}$ agonist; LAMA, long-acting muscarinic antagonist.

Table 4 GLMM model to evaluate the association between treatment and changes in HADS, anxiety, and depression scores

\begin{tabular}{|c|c|c|c|c|c|c|}
\hline Time & HADS score & Estimates & SE & $d f$ & $t$-value & $P$-value \\
\hline \multicolumn{7}{|c|}{ ICS/LABA use $\mathrm{e}^{\mathrm{a}}$} \\
\hline \multirow[t]{3}{*}{4 weeks } & Total score & -0.163 & 0.522 & 144 & -0.31 & 0.755 \\
\hline & Anxiety & $-0.08 \mathrm{I}$ & 0.287 & 144 & -0.28 & 0.778 \\
\hline & Depression & -0.123 & 0.298 & 144 & $-0.4 \mathrm{I}$ & 0.681 \\
\hline \multirow[t]{3}{*}{12 weeks } & Total score & -0.083 & 0.587 & $|3|$ & -0.14 & 0.888 \\
\hline & Anxiety & -0.008 & 0.311 & 131 & -0.03 & 0.980 \\
\hline & Depression & $-0.08 \mathrm{I}$ & 0.358 & $13 \mid$ & -0.23 & 0.820 \\
\hline \multicolumn{7}{|l|}{ LAMA $\mathrm{se}^{\mathrm{b}}$} \\
\hline \multirow[t]{3}{*}{4 weeks } & Total score & 0.772 & 0.598 & 129 & 1.29 & 0.199 \\
\hline & Anxiety & 0.419 & 0.319 & 129 & 1.32 & 0.191 \\
\hline & Depression & 0.365 & 0.352 & 129 & 1.04 & 0.301 \\
\hline \multirow[t]{3}{*}{12 weeks } & Total score & 0.587 & 0.678 & 117 & 0.87 & 0.389 \\
\hline & Anxiety & 0.591 & 0.355 & 117 & 1.67 & 0.098 \\
\hline & Depression & 0.010 & 0.410 & 117 & 0.02 & 0.980 \\
\hline
\end{tabular}

Notes: adjusted for marriage status and initial scores. ${ }^{\mathrm{b}}$ Adjusted for sex, BMI, FEV , smoking status, period of secondhand smoke exposure (year), and initial scores.

Abbreviations: $\mathrm{FEV}_{1}$, forced expiratory volume in I second; GLMM, general linear mixed model; HADS, Hospital Anxiety and Depression Scale; ICS, inhaled corticosteroid; LABA, long-acting $\beta_{2}$ agonist; LAMA, long-acting muscarinic antagonist; SE, standard error; BMI, body mass index; $d f$, degrees of freedom. 
more frequent exacerbation, ${ }^{31}$ higher in-hospital mortality, ${ }^{32}$ and decreased adherence to treatment in COPD patients. ${ }^{33}$ However, it has rarely been investigated whether inhaled drugs actually have an impact on mood problems among COPD patients, although studies have reported positive effects of pulmonary rehabilitation for COPD on mood disorders. ${ }^{34-36}$ Our results showed some improvement numerically in HADS scores in patients who were treated by ICS/LABA or LAMA, but we did not observe statistical significance. In the univariate analysis, those who were treated by ICS/LABA or LAMA showed a significant increase in HADS-A at the 4-week evaluation, but the statistical significance was lost in the multivariable analysis. LAMAs increased the HADS score by $1.83 \pm 4.86$ from baseline to 3 months during treatment, reaching the minimal clinically important difference in COPD patients. Puhan et a $\mathrm{l}^{37}$ evaluated 88 COPD patients, and reported that the minimal important difference was 1.40 for the HADS depression score, 1.32 for the HADS anxiety score, and 1.17 for the HADS total score based on the effect size approach. However, we did not find statistically significant differences in the proportion of patients in each group who meet the criteria for a minimal important difference in total HADS, HADS-A, and HADS-D. These results might be due to the small number of patients included.

The neutral effects of inhaled drugs on mood problems in our study could be explained by several reasons. First, the effects of these inhaled drugs may actually be minimal. For example, inhaled drugs might not readily cross the blood-brain barrier. ${ }^{38}$ The number of reports concerning psychiatric disorders was lower in those who received inhaled budesonide compared to the placebo group in a large clinical trial in asthma patients. ${ }^{39}$ A pooled analysis, including 9,149 tiotropium users and 7,865 placebo controls, showed no statistically significant difference in nervous system disorders. ${ }^{40}$ Second, the small number of participants might lead to a lack of statistical power. However, in the multivariate model, all of the $P$-values of the effects of ICS/LABA on the HADS score were $>0.681$, and all of the coefficients of the effects of LAMAs on the HADS score were positive values, indicating that LAMAs worsened the HADS score numerically, suggesting that these inhaled drugs were not associated with the trend to improve the HADS score.

Limitations of our study include the following: 1) the study was conducted in a real-world clinical practice without any intervention, possibly providing uncontrolled differences in characteristics between the intervention and control groups; 2) 3 months were short for the follow-up duration, considering most psychiatric diagnoses and management changes are based on a 6-month follow-up period; ${ }^{41} 3$ ) no evaluation of patient health status was performed at each visit, including symptoms, quality of life, exercise capacity, and lung functions, which might be confounding factors in the development of anxiety and/or depression; and 4) the actual adherence to inhaled therapies, which could affect drug efficacy, disease control, and symptoms, could not be evaluated.

In conclusion, our prospective observational study found no significant effects of inhaled treatment on mood problems among newly diagnosed COPD patients.

\section{Acknowledgments}

This study was funded by the National Evidence-based Healthcare Collaborating Agency (NECA; project number NC2011-004). The English in this document has been checked by at least two professional editors, both native speakers of English.

\section{Author contributions}

Min Kyung Hyun planned the study and wrote the manuscript. $\mathrm{Na}$ Rae Lee and Eun Jin Jang analyzed data. Jae-Joon Yim performed the survey. Chang-Hoon Lee planned the study, performed the survey, and wrote the manuscript. All authors contributed toward data analysis, drafting and critically revising the paper, gave final approval of the version to be published, and agree to be accountable for all aspects of the work.

\section{Disclosure}

The authors report no conflicts of interest in this work.

\section{References}

1. Global Strategy for the Diagnosis MaPoC. Global Initiative for Chronic Obstructive Lung Disease (GOLD). 2015. Available from: http://www. goldcopd.org/. Accessed February 12, 2016.

2. Calverley PM, Anderson JA, Celli B, et al. Salmeterol and fluticasone propionate and survival in chronic obstructive pulmonary disease. $N$ Engl J Med. 2007;356(8):775-789.

3. Celli BR, Thomas NE, Anderson JA, et al. Effect of pharmacotherapy on rate of decline of lung function in chronic obstructive pulmonary disease: results from the TORCH study. Am J Respir Crit Care Med. 2008; 178(4):332-338.

4. Tashkin DP, Celli B, Senn S, et al. A 4-year trial of tiotropium in chronic obstructive pulmonary disease. $N$ Engl J Med. 2008;359(15): 1543-1554.

5. Suissa S, Kezouh A, Ernst P. Inhaled corticosteroids and the risks of diabetes onset and progression. Am J Med. 2010;123(11):1001-1006.

6. Richy F, Bousquet J, Ehrlich GE, et al. Inhaled corticosteroids effects on bone in asthmatic and COPD patients: a quantitative systematic review. Osteoporos Int. 2003;14(3):179-190.

7. Weatherall M, James K, Clay J, et al. Dose-response relationship for risk of non-vertebral fracture with inhaled corticosteroids. Clin Exp Allergy. 2008;38(9):1451-1458.

8. Weatherall M, Wijesinghe M, Perrin K, Harwood M, Beasley R. Metaanalysis of the risk of mortality with salmeterol and the effect of concomitant inhaled corticosteroid therapy. Thorax. 2010;65(1):39-43. 
9. Singh S, Loke YK, Furberg CD. Inhaled anticholinergics and risk of major adverse cardiovascular events in patients with chronic obstructive pulmonary disease: a systematic review and meta-analysis. JAMA. 2008; 300(12):1439-1450.

10. Naka T, Ide $S$, Nakako $T$, et al. Activation of beta-adrenoceptors in the bed nucleus of the stria terminalis induces food intake reduction and anxiety-like behaviors. Neuropharmacology. 2013;67:326-330.

11. Mineur YS, Obayemi A, Wigestrand MB, et al. Cholinergic signaling in the hippocampus regulates social stress resilience and anxiety- and depression-like behavior. Proc Natl Acad Sci U S A. 2013;110(9): 3573-3578.

12. Dagyte G, Den Boer JA, Trentani A. The cholinergic system and depression. Behav Brain Res. 2011;221(2):574-582.

13. Drevets WC, Zarate CA Jr, Furey ML. Antidepressant effects of the muscarinic cholinergic receptor antagonist scopolamine: a review. Biol Psychiatry. 2013;73(12):1156-1163.

14. Kenna HA, Poon AW, de los Angeles CP, Koran LM. Psychiatric complications of treatment with corticosteroids: review with case report. Psychiatry Clin Neurosci. 2011;65(6):549-560.

15. Brown ES. Effects of glucocorticoids on mood, memory, and the hippocampus. Treatment and preventive therapy. Ann N Y Acad Sci. 2009; 1179:41-55.

16. Zigmond AS, Snaith RP. The hospital anxiety and depression scale. Acta Psychiatr Scand. 1983;67(6):361-370.

17. Choi SK, Min SJ, Cho MS, Joung H, Park SM. Anxiety and depression among North Korean young defectors in South Korea and their association with health-related quality of life. Yonsei Med J. 2011;52(3):502-509.

18. Kang HJ, Stewart R, Kim JM, et al. Comparative validity of depression assessment scales for screening poststroke depression. J Affect Disord. 2013;147(1-3):186-191.

19. Jang JE, Kim SW, Kim SY, et al. Religiosity, depression, and quality of life in Korean patients with breast cancer: a 1-year prospective longitudinal study. Psychooncology. 2013;22(4):922-929.

20. Global Initiative for Chronic Obstructive Lung Disease. Global strategy for the diagnosis, management, and prevention of chronic obstructive pulmonary disease. 2014. Available from: http://www.goldcopd.org/ guidelines-global-strategy-for-diagnosis-management.html. Accessed March 18, 2016.

21. Snaith RP. The hospital anxiety and depression scale. Health Qual Life Outcomes. 2003;1:29.

22. Richmond M, Seger D. Central anticholinergic syndrome in a child: a case report. J Emerg Med. 1985;3(6):453-456.

23. Frielle T, Daniel KW, Caron MG, Lefkowitz RJ. Structural basis of beta-adrenergic receptor subtype specificity studied with chimeric beta 1/beta 2-adrenergic receptors. Proc Natl Acad Sci U S A. 1988; 85(24):9494-9498.

24. Gupta P, O'Mahony MS. Potential adverse effects of bronchodilators in the treatment of airways obstruction in older people: recommendations for prescribing. Drugs Aging. 2008;25(5):415-443.

25. Johnson M. The $\beta$-adrenoceptor. Am J Respir Crit Care Med. 1998; 158(5 Pt 3):S146-S153.

26. van Vlymen JM, Parlow JL. The effects of reversal of neuromuscular blockade on autonomic control in the perioperative period. Anesth Analg. 1997;84(1):148-154.
27. Singh S, Loke YK, Enright P, Furberg CD. Pro-arrhythmic and proischaemic effects of inhaled anticholinergic medications. Thorax. 2013; 68(1):114-116

28. Gift AG, Wood RM, Cahill CA. Depression, somatization and steroid use in chronic obstructive pulmonary disease. Int J Nurs Stud. 1989; 26(3):281-286.

29. Maurer J, Rebbapragada V, Borson S, et al. Anxiety and depression in COPD: current understanding, unanswered questions, and research needs. Chest. 2008;134(4 Suppl):43S-56S.

30. Postolache P, Costin M, Dumbrava EL, Cojocaru DC. Anxiety and depression in patients with chronic obstructive pulmonary disease: an open agenda for research. Rev Med Chir Soc Med Nat Iasi. 2014;118(1): $39-44$.

31. Xu W, Collet JP, Shapiro S, et al. Independent effect of depression and anxiety on chronic obstructive pulmonary disease exacerbations and hospitalizations. Am J Respir Crit Care Med. 2008;178(9):913-920.

32. Ng TP, Niti M, Tan WC, Cao Z, Ong KC, Eng P. Depressive symptoms and chronic obstructive pulmonary disease: effect on mortality, hospital readmission, symptom burden, functional status, and quality of life. Arch Intern Med. 2007;167(1):60-67.

33. Turan O, Yemez B, Itil O. The effects of anxiety and depression symptoms on treatment adherence in COPD patients. Prim Health Care Res Dev. 2014;15(3):244-251.

34. Harrison SL, Greening NJ, Williams JE, Morgan MD, Steiner MC, Singh SJ. Have we underestimated the efficacy of pulmonary rehabilitation in improving mood? Respir Med. 2012;106(6):838-844.

35. Coventry PA. Does pulmonary rehabilitation reduce anxiety and depression in chronic obstructive pulmonary disease? Curr Opin Pulm Med. 2009;15(2):143-149.

36. Paz-Diaz H, Montes de Oca M, Lopez JM, Celli BR. Pulmonary rehabilitation improves depression, anxiety, dyspnea and health status in patients with COPD. Am J Phys Med Rehabil. 2007;86(1):30-36.

37. Puhan MA, Frey M, Buchi S, Schunemann HJ. The minimal important difference of the hospital anxiety and depression scale in patients with chronic obstructive pulmonary disease. Health Qual Life Outcomes. 2008;6:46.

38. Heredia JL. Tiotropium bromide: an update. Open Respir Med J. 2009; $3: 43-52$.

39. Sheffer AL, Silverman M, Woolcock AJ, Diaz PV, Lindberg B, Lindmark B. Long-term safety of once-daily budesonide in patients with early-onset mild persistent asthma: results of the Inhaled Steroid Treatment as Regular Therapy in Early Asthma (START) study. Ann Allergy Asthma Immunol. 2005;94(1):48-54.

40. Kesten S, Celli B, Decramer M, Leimer I, Tashkin D. Tiotropium HandiHaler in the treatment of COPD: a safety review. Int J Chron Obstruct Pulmon Dis. 2009;4:397-409.

41. NICE. Depression: Evidence Update April 2012. National Institute for Health and Clinical Excellence; 2012. Available from: http://www.nice. org.uk/guidance/cg90/evidence. Accessed February 12, 2016.
International Journal of COPD

\section{Publish your work in this journal}

The International Journal of COPD is an international, peer-reviewed journal of therapeutics and pharmacology focusing on concise rapid reporting of clinical studies and reviews in COPD. Special focus is given to the pathophysiological processes underlying the disease, intervention programs, patient focused education, and self management protocols.

\section{Dovepress}

This journal is indexed on PubMed Central, MedLine and CAS. The manuscript management system is completely online and includes a very quick and fair peer-review system, which is all easy to use. Visit http://www.dovepress.com/testimonials.php to read real quotes from published authors. 